\title{
Genome-wide association mapping for heading date in oats under subtropical
}

\section{environments}

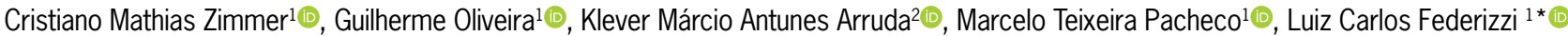

\author{
UUniversidade Federal do Rio Grande do Sul/Faculdade \\ de Agronomia - Depto. de Plantas de Lavoura, Av. Bento \\ Gonçalves, 7712 - 91540-000 - Porto Alegre, RS - Brasil. \\ Instituto Agronômico do Paraná, Área de Melhoramento e \\ Genética Vegetal, Rod. Celso Garcia Cid, 375 - 86047-902 \\ - Londrina, PR - Brasil. \\ *Corresponding author <federizi@ufrgs.br>
}

Edited by: Alencar Xavier

Received November 16, 2020

Accepted January 20, 2021

\begin{abstract}
Selection for heading date has been a decisive factor to increase areas cropped with oats in Brazil. Although important to oat breeders, genomic regions controlling heading date have not been completely identified. The objective of this study was to identify genomic regions controlling oat heading date in subtropical environments. A set of 412 oat genotypes, developed from 1974 to 2015, was assessed for heading date in contrasting environments and genotyped using genotyping-by-sequencing (GBS). Phenotypic and genotypic data were used in single and multi-environment association models. Quantitative trait loci (QTL) associated to heading date were identified on oat consensus groups Mrg02, Mrg05, Mrg06, Mrg12, and Mrg21. Some of the findings confirmed the association of genomic regions with heading date, while others emerge as new candidate regions associated to the trait. The genomic regions identified on Mrg02 and Mrg12 were associated to Vernalization $3(\mathrm{Vrn} 3)$, while the genomic region identified on Mrg21 is associated with Vernalization 1 ( Vrn1). The Vrn1 region was detected in Londrina, an environment with reduced vernalization condition, and in the multi-environment model. The results reveal that some genotypes of the panel are responsive to vernalization, increasing the days to heading without this environmental stimulus. Our results provide important contribution for a better understanding of heading date in subtropical environments and a strong basis for marker-assisted selection in oats.
\end{abstract}

Keywords: Avena sativa L., flowering time, marker-assisted selection, quantitative trait loci

\section{Introduction}

Heading date is one of the most important traits for selection in oats (Avena sativa L.). In southern Brazil, adaptation to subtropical environments is the first selection criteria of oat breeders, mainly focusing on flowering time adequacy and disease resistance. After many rounds of selections, varieties are developed combining earliness, resistance to different pathogens, and high yield potential. Although a significant genetic gain has been achieved over the last decades, the genetic basis involving oat adaptation to subtropical environments has not been fully understood.

Many studies have aimed to identify genomic regions associated to heading date in oats, which represents an important step to implement markerassisted selection (MAS) and accelerate genetic gain. Studying two biparental populations derived from naked by hulled crosses, Zimmer et al. (2018) identified three major quantitative trait loci (QTL) controlling heading date in oats. One of these QTL was associated to the SNP marker GMI_ES_LB_11316 on Mrg02 (Zimmer et al., 2018). This QTL was first detected by Esvelt Klos et al. (2016) in a genome-wide association study (GWAS) involving a large oat panel assessed across 15 locationsyears. The Vernalization 3 (Vrn3) and CONSTANS (CO) were suggested as candidate genes controlling heading date on Mrg02 (Esvelt Klos et al., 2016).

The identification of QTL associated with oat heading date in subtropical environments has been performed exclusively using biparental mapping populations. Some of these QTL have major limitations to be applied in oat breeding programs, including: i) restricted allelic diversity segregating between parental lines; and ii) mapping resolution that may be limited due to reduced recombination events being captured. In this context, GWAS emerges as an alternative approach to overcome these pitfalls using ancestral recombination to improve mapping resolution and accessing alleles from more than two individuals. GWAS has been applied to identify genomic regions controlling important traits in oats, including $\beta$-glucan content (Newell et al., 2012; Asoro et al., 2013; Zimmer et al., 2020), crown rust resistance (Esvelt Klos et al., 2017; McNish et al., 2020), and heading date (Esvelt Klos et al., 2016). The objective of this study was to identify genomic regions controlling oat heading date in subtropical environments by performing a GWAS using a panel of 412 unrelated diverse genotypes.

\section{Materials and Methods}

\section{Plant material}

We assessed 412 inbred lines for heading date. These genotypes represent the Federal University of Rio Grande do Sul (UFRGS) Oat Panel, which was assembled with a priority placed on maximizing the genetic diversity (Zimmer et al., 2020). The UFRGS Oat Panel was originally composed by 384 hulled genotypes 
and 29 naked genotypes from different oat breeding programs. The UFRGS Oat Breeding Program developed 360 genotypes from 1974 to 2015, 14 genotypes were developed by other Brazilian oat breeding programs, and 39 were developed by foreign oat breeding programs, mainly from North America.

\section{Field experiments and heading date assessment}

The experiments were carried out in two environments in southern Brazil. In 2017, the UFRGS Oat Panel was assessed in the municipality of Londrina $\left(23^{\circ} 21^{\prime}\right.$ $\mathrm{S}, 51^{\circ} 10^{\prime} \mathrm{W}$, altitude $\left.548 \mathrm{~m}\right)$, Paraná State. The experiment was conducted in a randomized complete block design with two replicates. Each experimental unit was composed by a single $1 \mathrm{~m}$ long row spaced $0.25 \mathrm{~m}$ apart. Planting was performed manually at a rate of 50 and 60 seeds per plot for hulled and naked genotypes, respectively. Base fertilization was $275 \mathrm{~kg}$ $\mathrm{ha}^{-1}$ of a $5-20-20 \mathrm{~N}-\mathrm{P}-\mathrm{K}$ formula. Topdressing $\mathrm{N}$, in the form of dry urea, was applied once, when plants showed four extended leaves, at a rate of $20 \mathrm{~kg} \mathrm{ha}^{-1}$ of N. In 2018, the UFRGS Oat Panel was assessed in Eldorado do Sul $\left(30^{\circ} 07^{\prime} \mathrm{S}, 51^{\circ} 41^{\prime} \mathrm{W}\right.$, altitude 70 $\mathrm{m})$, Rio Grande do Sul State. The experiment was conducted in a randomized complete block design with two replicates. Each experimental unit was composed by two rows, $2 \mathrm{~m}$ long each, spaced $0.2 \mathrm{~m}$ apart, and $0.4 \mathrm{~m}$ between experimental units. Planting was carried out mechanically at a rate of 120 seeds per row. Base fertilization was $300 \mathrm{~kg} \mathrm{ha}^{-1}$ of a 5-30-15 $\mathrm{N}-\mathrm{P}-\mathrm{K}$ formula, and topdressing $\mathrm{N}$, in the form of dry urea, was applied twice, when plants showed three and six extended leaves, at a rate of $33 \mathrm{~kg} \mathrm{ha}^{-1}$ of $\mathrm{N}$ per application. Pests, fungal diseases, and weeds were chemically controlled in both experiments.

Londrina is characterized by high temperature averages and low precipitation rates during the oat crop season, while Eldorado do Sul shows moderate temperatures and high precipitation rates during the oat crop season. In this sense, these contrasting environments represent a broad range of the Brazilian subtropical environments. Heading date was assessed as the number of days from plant emergence in both locations until the moment when $50 \%$ of the plants showed panicles emerged from the main stem (stage 55 from Zadoks' scale; Zadoks et al., 1974) in each experimental unit.

\section{DNA isolation and genotyping-by-sequence}

DNA was isolated from freeze-dried tissue collected from seedlings of a single panicle of each genotype at the University of Minnesota Genomics Center (UMGC). After quantification, genotyping-by-sequencing (GBS) was carried out at UMGC, using a two-enzyme (PstI and MspI) protocol adapted from Poland et al. (2012), as described by Zimmer et al. (2020). Briefly, libraries were sequenced using a NovaSeq ${ }^{\mathrm{TM}} 6000$ sequencer (Illumina, San Diego, USA), producing individual 100bp single-end reads. Sequence data was trimmed using Trimmomatic (Bolger et al., 2014) and quality control of readings was verified using FastQC (Andrews, 2010).

\section{SNP calling and filtering}

Single-nucleotide polymorphisms (SNP) were called using Haplotag (Tinker et al., 2016), aligned to the oat consensus map (Bekele et al., 2018), and filtered as described by Zimmer et al. (2020). After the filtering steps, 10,209 SNP were kept in the final genotypic matrix. The missing data were imputed using the 'expectationmaximization' (EM) method with the 'A.mat' function from the rrBLUP (Endelman, 2011) package in the $\mathrm{R}$ software.

\section{Genome-wide association mapping for heading date}

GWAS was carried out for each trial separately and also considering a multi-environment model. The analyses were performed using the 'GWAS' function from the rrBLUP (Endelman, 2011) R package, accounting for population structure and kinship $(\mathrm{Q}+\mathrm{K}$ model), based on Yu et al. 2006, as follow:

$y=X \beta+Z g+S \tau+\varepsilon$

where: $y$ is a vector of phenotypic variation; $\beta$ is a vector of fixed effects that can model environmental factors and the population structure; $g$ models the genetic background as a random effect; $\tau$ models the additive SNP effect as a fixed effect; and $\varepsilon$ is a vector of residual effects.

The population structure was accounted for using the first three principal components, following Zimmer et al. (2020). Relatedness was accounted using a kinship matrix calculated using the 'A.mat' function from the rrBLUP (Endelman, 2011) R package. The population structure and location were fit as fixed effect. Observed versus expected $p$-values under the null hypothesis were visualized for each model to verify signs of statistical inflation. Two statistical significance thresholds were considered: i) Bonferroni ( $\left.p \leq 4.90^{-06}\right)$; and ii) a false discovery rate (FDR) described by Benjamini and Hochberg (1995). Both significance thresholds considered $\alpha=0.05$.

\section{Statistical analysis}

The analysis of variance (ANOVA) was performed to identify differences among genotypes, between environments, and for the genotype by environment interaction, as follow:

$y=\mu+G+E+G E+\varepsilon$ 
where: $y$ is the trait mean (heading date); $\mu$ is the grand mean; $G$ is the genotype; $E$ is the environment; $G E$ is the genotype by environment interaction; and $\varepsilon$ is the experimental error. The ANOVA was carried out using PROC GLM in SAS (Statistical Analysis System, version 9.4).

\section{Results}

\section{Phenotypic variation for heading date in contrasting subtropical environments.}

The UFRGS Oat Panel showed a wide phenotypic variation for heading date in both environments. In Londrina, the number of days to heading ranged from 54 to 124 days among genotypes, with an average of 74 days (Figure 1). In Eldorado do Sul, days to heading varied from 70 to 113 days among

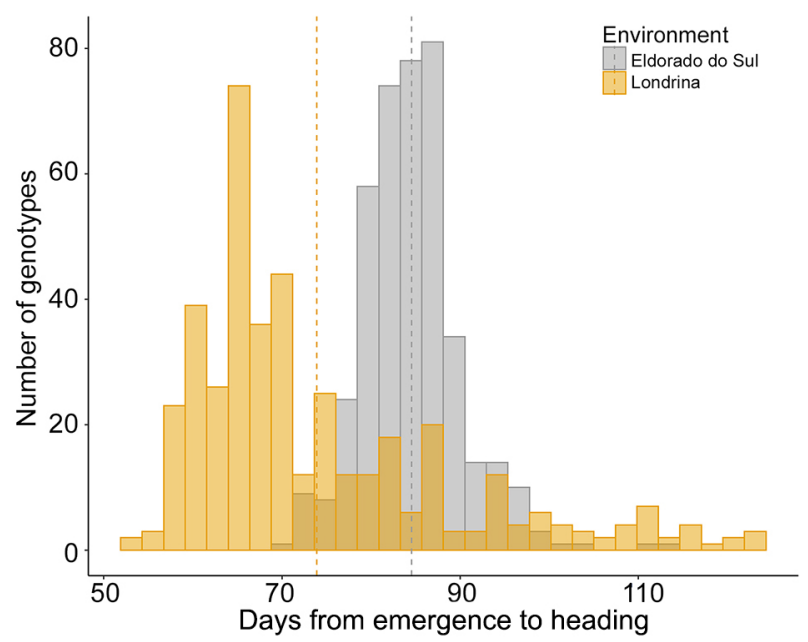

Figure 1 - Phenotypic variation for oat heading date in two subtropical environments. Dashed lines represent overall mean of each environment.

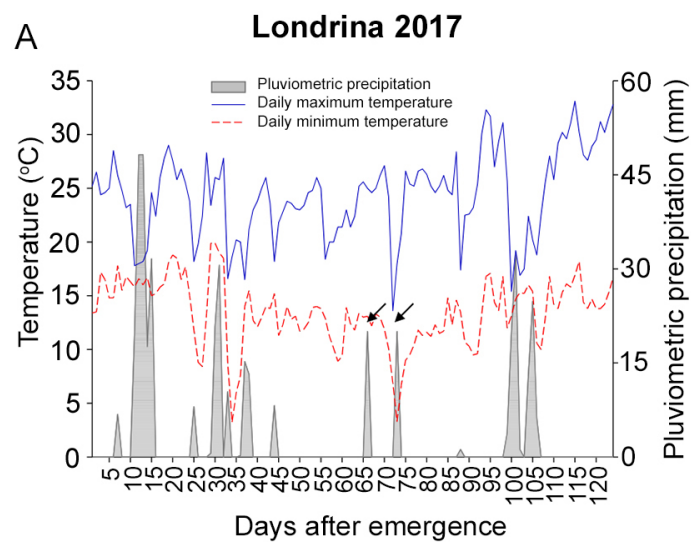

genotypes, with an average of 84 days (Figure 1). Environmental stimuli recorded during the season are shown in Figure 2, illustrating the contrasting data between Londrina (Figure 2A) and Eldorado do Sul (Figure 2B). Differences for heading date were identified among genotypes and between environments, as well as for the interaction between genotype and environment.

\section{Genomic regions associated with heading date in each environment}

Three and four QTL associated with heading date were identified in Londrina and Eldorado do Sul, respectively (Figures 3A and 3B). In Londrina, the QTL influencing heading date were located on the linkage groups Mrg02, Mrg05, and Mrg21 of the oat consensus map (Figure 3B). The QTL located on Mrg02 was associated with SNP markers avgbs_95303.1.18, avgbs_95272.1.27, and avgbs2_89121.1.55, covering a genetic region of $0.5 \mathrm{cM}$ (Table 1). The QTL detected on Mrg05 is associated with the marker avgbs_110804.1.56, which is located at the position $56.5 \mathrm{cM}$ (Table 1). On the consensus linkage group Mrg21, the QTL influencing heading date is associated to markers avgbs_cluster_16378.1.28, avgbs2_188948.1.42, avgbs_119304.1.27, avgbs2_165679.1.58, and avgbs_93789.1.22, located at position $122.8 \mathrm{cM}$ (Table 1).

Four QTL affecting heading date were detected in Eldorado do Sul. Two of these QTL were located on the linkage group Mrg02, while the others were located on the linkage groups Mrg05 and Mrg12 of the oat consensus map (Figure 3A). The first QTL identified on Mrg02 is associated to marker avgbs_95272.1.27, located at position $34.1 \mathrm{cM}$. The second QTL was associated to marker avgbs2_17557.1.22, mapped at position $87.3 \mathrm{cM}$ in the same consensus group. The QTL on Mrg05 is associated to marker avgbs_110804.1.56, mapped at position $56.5 \mathrm{cM}$. On Mrg12, the QTL influencing heading date was associated to marker avgbs2_169922.1.31, located at position $44.5 \mathrm{cM}$.

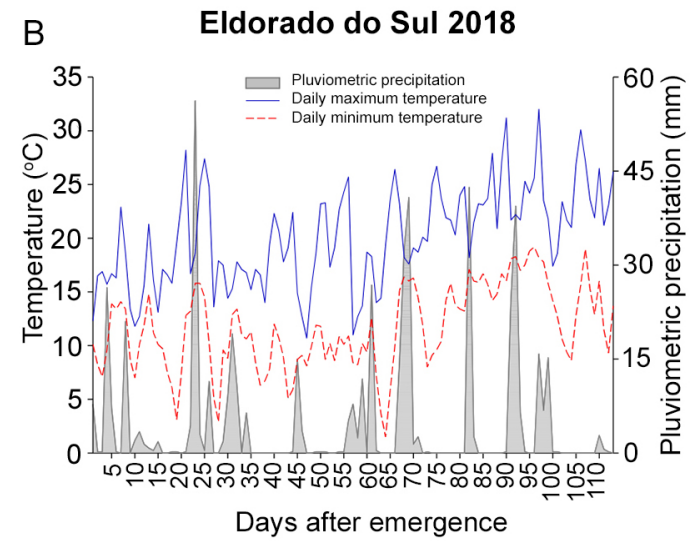

Figure 2 - Environmental conditions in Londrina (A) and Eldorado do Sul (B) from planting to heading date. Black arrows represent irrigation. 


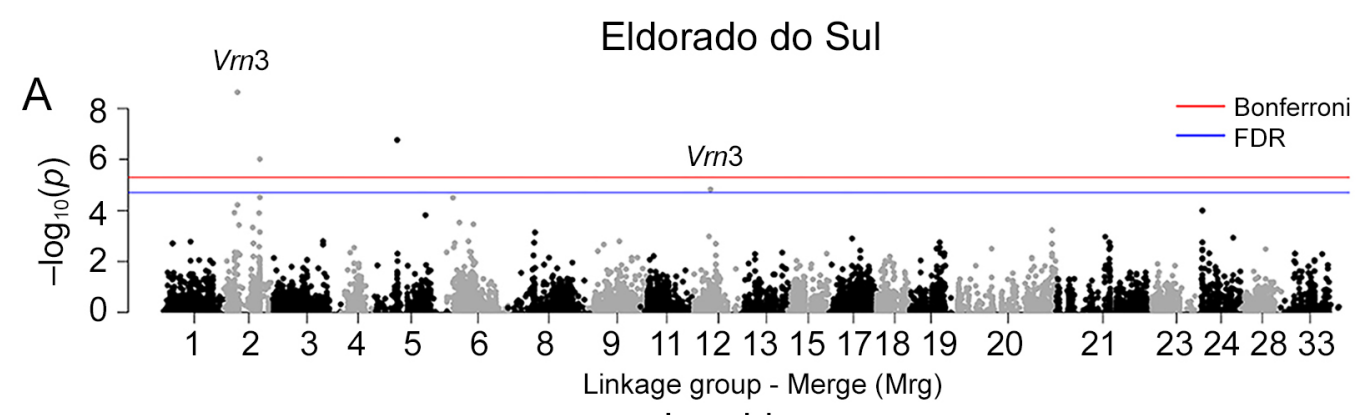

Londrina
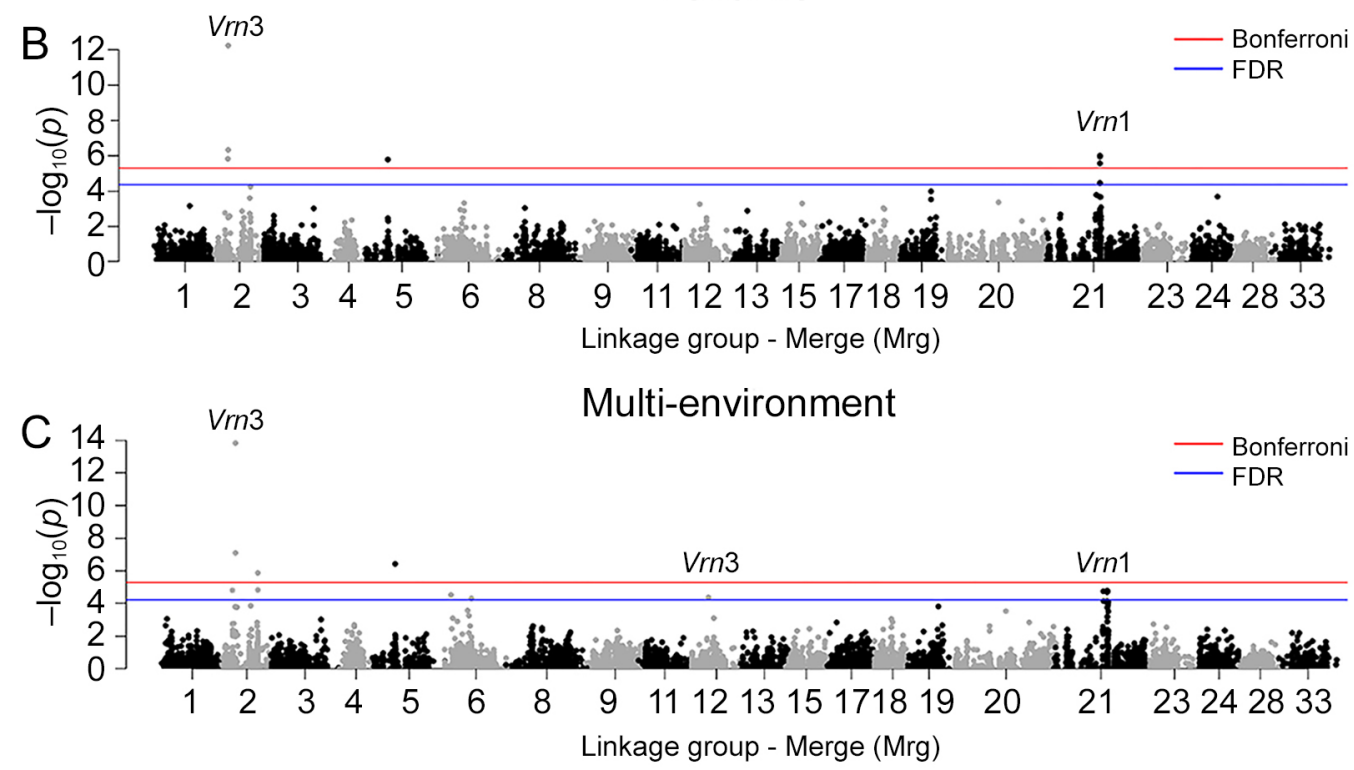

Figure 3 - Manhattan plots for markers associated to heading date in oats. The red horizontal line represents the Bonferroni threshold while the blue line represents the false discovery rate (FDR) of $5 \%$. A - Eldorado do Sul; B - Londrina; C - Multi-environment model.

Table 1 - Markers influencing oat heading date in subtropical environments.

\begin{tabular}{|c|c|c|c|c|c|c|c|c|}
\hline Marker & $\begin{array}{c}\text { Consensus } \\
\mathrm{LG}^{\ddagger}\end{array}$ & Position ${ }^{\ddagger}$ & $\begin{array}{c}\text { LON } \\
-\log _{10}(p)\end{array}$ & $\begin{array}{c}\text { ELD } \\
-\log _{10}(p)\end{array}$ & $\begin{array}{c}\text { Multi-e } \\
-\log _{10}(p)\end{array}$ & PVE & $\begin{array}{l}\text { Candidate } \\
\text { gene }\end{array}$ & Reference \\
\hline & & $\mathrm{cM}$ & & & & $\%$ & & \\
\hline avgbs2_82665.2.9 & Mrg02 & 26.8 & - & - & 4.81 & 4.17 & & \\
\hline avgbs_95303.1.18 & Mrg02 & 33.6 & 5.84 & - & - & 5.24 & Vrn3 & Esvelt Klos et al. (2016) \\
\hline avgbs_95272.1.27 & Mrg02 & 34.1 & 12.25 & 8.65 & 13.82 & 13.22 & Vrn3 & Esvelt Klos et al. (2016) \\
\hline avgbs2_89121.1.55 & Mrg02 & 34.1 & 6.33 & - & 7.09 & 6.52 & Vrn3 & Esvelt Klos et al. (2016) \\
\hline avgbs2_17557.1.22 & Mrg02 & 87.3 & - & 6.02 & 5.86 & 5.42 & & \\
\hline avgbs2_67891.1.49 & Mrg02 & 87.3 & - & - & 4.82 & 4.19 & & \\
\hline avgbs_110804.1.56 & Mrg05 & 56.5 & 5.80 & 6.77 & 6.44 & 6.19 & & \\
\hline avgbs_220423.1.53 & Mrg06 & 14.5 & - & - & 4.54 & 3.90 & & \\
\hline avgbs_cluster_42139.1.55 & Mrg06 & 63.2 & - & - & 4.31 & 3.66 & & \\
\hline avgbs2_169922.1.31 & Mrg12 & 44.5 & - & 4.84 & 4.37 & 4.21 & Vrn3 & Nava et al. (2012) \\
\hline avgbs2_71505.1.33 & Mrg21 & 113.2 & - & - & 4.73 & 4.09 & Vrn1 & Maloney et al. (2011) \\
\hline avgbs_cluster_16378.1.28 & Mrg21 & 122.8 & 6.01 & - & 4.78 & 5.41 & & \\
\hline avgbs2_188948.1.42 & Mrg21 & 122.8 & 5.94 & - & - & 5.34 & & \\
\hline avgbs_119304.1.27 & Mrg21 & 122.8 & 5.56 & - & 4.69 & 4.95 & & \\
\hline avgbs2_165679.1.58 & Mrg21 & 122.8 & 4.46 & - & - & 3.81 & & \\
\hline avgbs_93789.1.22 & Mrg21 & 122.8 & 4.44 & - & - & 3.79 & & \\
\hline
\end{tabular}

$\mathrm{cM}$ = centiMorgans; $\mathrm{LG}$ = linkage group; $\mathrm{LON}=$ Londrina; $\mathrm{ELD}=$ Eldorado do Sul; PVE = variance explained by the single-nucleotide polymorphism (SNP); ${ }^{\mathrm{B}} \mathrm{Based}$ on Bekele et al. (2018). 


\section{Genomic regions associated to heading date in the} multi-environment model

Seven QTL were detected influencing heading date in the multi-environment model (Figure 3C). These QTL were identified on Mrg02, Mrg05, Mrg06, Mrg12, and Mrg21 linkage groups of the oat consensus map, with Mrg02 and Mrg06 showing two QTL each. The first QTL located on Mrg02 was associated with SNP markers avgbs2_82665.2.9, avgbs_95272.1.27, and avgbs2_89121.1.55, covering a genetic region of 7.3 cM (Table 1). SNP marker avgbs2_82665.2.9 is mapped at position $26.8 \mathrm{cM}$, while the others are mapped at position $34.1 \mathrm{cM}$ in the Mrg02 linkage group. The second QTL detected on Mrg02 is associated to SNP markers avgbs2_17557.1.22 and avgbs2_67891.1.49, located at position $87.3 \mathrm{cM}$ (Table 1). The QTL detected on Mrg05 linkage group is associated to avgbs_110804.1.56, mapped at position $56.5 \mathrm{cM}$ (Table 1). On the Mrg06 linkage group, the QTL controlling heading date are associated to SNP markers avgbs_220423.1.53 and avgbs_cluster_42139.1.55, located at positions 14.5 and $63.2 \mathrm{cM}$, respectively. The QTL detected on Mrg12 was associated to marker avgbs2_169922.1.31, mapped at position 44.5 cM. Markers avgbs2_71505.1.33, avgbs_cluster_16378.1.28, and avgbs_119304.1.27 were associated to the QTL detected on the consensus linkage group Mrg21, covering a genetic region of $9.6 \mathrm{cM}$. Marker avgbs2_71505.1.33 is mapped at position 113.2 cM on Mrg21, while the others are mapped at position $122.8 \mathrm{cM}$ (Table 1).

The loci detected on Mrg02 (avgbs_95272.1.27) and on Mrg05 (avgbs_110804.1.56) were detected in both environments, as well as in the multi-environment model, suggesting high relevance of these genomic regions on heading date in subtropical environments. The second QTL detected on Mrg02 and the QTL detected on Mrg12 were identified only in Eldorado do Sul and in the multi-environment model. Similarly, the locus located on Mrg21 was detected only in Londrina and in the multi-environment model. Conversely, the two loci detected on Mrg06 consensus group were identified only in the multi-environment model. The Q-Q plots show that the relationship between the observed and expected $p$-values is closely aligned with expectations under the null hypothesis, without any signal of statistical inflation, regardless of the model applied (Figures 4A-4C).

\section{Discussion}

In this study, we assessed a genetic diverse oat panel for heading date in two subtropical environments. Phenotypic and genotypic data were used in GWAS, based on single and multi-environment models, allowing the identification of QTL influencing heading date in oats.

The phenotypic data illustrate that the UFRGS Oat Panel show a wide variation for heading date, which is more pronounced in Londrina (Figure 1). The observed amplitude for heading date in Londrina, ranging from 54 to 124 days, reinforces that the UFRGS Oat Panel has potential for GWAS, as described by Zimmer et al. (2020). The UFRGS Oat Panel shows wider phenotypic variation for heading date than biparental populations previously studied in subtropical conditions. Studying two hulled by naked oat mapping populations, Zimmer et al. (2018) observed a phenotypic variation ranging from 70 to 103 days to heading date between recombinant inbred lines, varying according with planting date and mapping population. Characterizing heading date of $563 \mathrm{~F}_{2}$ individuals from a southern-by-spring oat population, Mazurkievicz et al. (2019) identified a phenotypic variation ranging from 79 to 132 days. In this sense, the wider phenotypic variation of the UFRGS Oat Panel, associated to a large set of markers, detected important genomic regions associated to heading date.

The GWAS showed that the most significant region associated to heading date is located on Mrg02 (Table 1), in both single and multi-environment models (Figures 3A-3C), explaining up to $13 \%$ of the phenotypic variation (Table 1). This region has been widely detected in other studies, in both biparental or unrelated mapping populations (Locatelli et al., 2006;
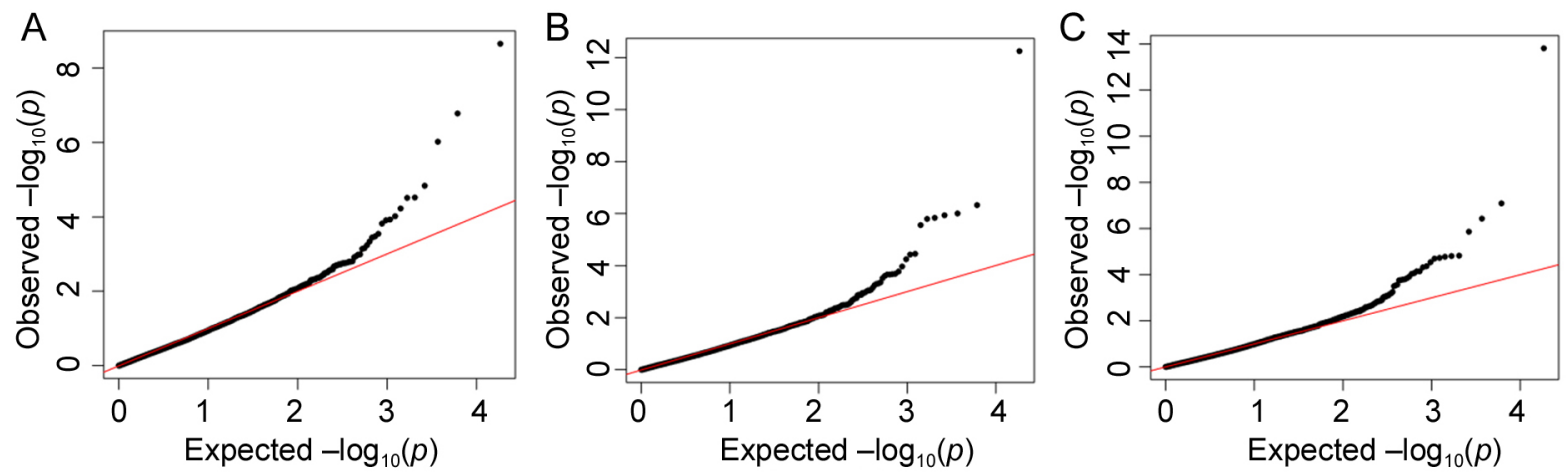

Figure 4 - Quantile-quantile plots of observed versus expected p-values under the null hypothesis for heading date in oats. A - Eldorado do Sul; B - Londrina; C - multi-environment model. 
Esvelt Klos et al., 2016; Zimmer et al., 2018; Sunstrum et al., 2019). Studying an oat panel of 682 lines across 15 locations-years in the United States, Canada, and the United Kingdom, Esvelt Klos et al. (2016) detected consistent effects on heading date between 29 and 34.1 $\mathrm{cM}$ on $\mathrm{Mrg02}$, overlapping the same genomic regions identified in our study (Table 1). The same genomic region was detected by Zimmer et al. (2018) in a naked by hulled oat mapping population, with the QTL peak near to SNP marker GMI_ES_LB_11316, explaining 20-22\% of the phenotypic variation for heading date, according to the planting date. Esvelt Klos et al. (2016) showed that marker GMI_ES_LB_11316, located at $34.1 \mathrm{cM}$ in the oat consensus map (Bekele et al., 2018), explains 18 $\%$ of the phenotypic variation on average, ranging from 12 to $28 \%$ according to the environmental condition. There is strong evidence that the QTL detected on $\mathrm{Mrg} 02$ (26.8 - $34.1 \mathrm{cM}$ ) corresponds to the major locus $\mathrm{Ma}-2$, as reported by Federizzi et al. (1996), and is associated to early heading date. This locus possibly corresponds to the locus Di1 identified by Locatelli et al. (2006) in two mapping populations involving the parental line UFRGS 8. Considered an important founder for modern oat varieties of the UFRGS Oat Breeding Program, UFRGS 8 is included in many pedigrees of the UFRGS Oat Panel. Di1 was mapped into a region syntenic to the linkage group 17 of the KO oat mapping population (O'Donoughue et al., 1995), corresponding to the linkage group Mrg02 of the current consensus oat map (Bekele et al., 2018). A QTL associated to heading date on the mapping population 'UTau/U017' (Zimmer et al., 2018), associated to marker GMI_ES_LB_11316 on Mrg02, revealed that alleles from the Brazilian variety URS Taura acts by decreasing days to heading, reinforcing that this region corresponds to locus $M a-2$, as described by Federizzi et al. (1996). Possibly, all markers associated to heading date on $\mathrm{Mrg} 02$ (26.8 - $34.1 \mathrm{cM}$ and $87.3 \mathrm{cM}$ ) corresponds to the same QTL. These regions on Mrg02 seem to be in high linkage disequilibrium, as revealed by Esvelt Klos et al. (2016), McNish et al. (2020), and Zimmer et al. (2020).

A QTL influencing heading date was also detected on Mrg12 (Table 1, Figures 3A and 3C). Candidate genes for heading date located on both Mrg02 and Mrg12 include Vrn3, which corresponds to HEADING DATE 1 (HD1) in rice and FLOWERING LOCUS T (FT) in Arabidopsis (Esvelt Klos et al., 2016). This is explained because Mrg02 and Mrg12 are suggested as homoeolog chromosomes (Chaffin et al., 2016) that originate from $\mathrm{D}$ and A genomes, respectively (Yan et al., 2016). Nava et al. (2012) cloned a partial ortholog of Vrn3 in oats and mapped it to the region of the KO map that corresponds to position $40.9 \mathrm{cM}$ on Mrg12, suggesting that this locus may be associated to vernalization and photoperiod. A comparative mapping study showed that marker GMI_ES_LB_11316 (34.1 cM on Mrg02) is highly conserved in oats, rice, and stiff brome, revealing nucleotide similarities greater than $86 \%$ between these species (Zimmer et al., 2018). On the rice genomic region flanked by oat markers, Zimmer et al. (2018) identified three important genes associated to heading date: HEADING DATE 3B (Hd3b)/RICE FLOWERING LOCUS T1 (RFT1), HEADING DATE $3 A(H d 3 a)$, and the MADS-box gene OsMADS5. These results reinforce that Vrn3 may be associated to the QTL identified on Mrg02 and Mrg12.

Late flowering of some genotypes in Londrina revealed a vernalization requirement for heading (Figure 1). Although Londrina showed a smaller average for days to heading, the number of genotypes with late heading was higher than in Eldorado do Sul (Figure 1). Genotypes with late heading in Londrina reduced the number of days to heading in Eldorado do Sul, where lower temperatures were recorded (Figure 2B), confirming that a vernalization gene might be involved. The QTL found on Mrg21 (six markers at 113.2 - 122.8 cM; Table 1) is associated to vernalization. An ortholog of the vernalization gene Vrn1 was mapped on KO (Nava et al., 2012) in a region that corresponds to the consensus group Mrg20 (Bekele et al., 2018). The Vrn1 gene was also suggested on Mrg21 (Esvelt Klos et al., 2016; Maloney et al., 2011). The QTL for vernalization response have been mapped on both $\operatorname{Mrg} 20$ and Mrg21 (Holland et al., 2002; Nava et al., 2012; Sunstrum et al., 2019). This is explained because Mrg20 and Mrg21 are homoeolog chromosomes from A and D genomes, respectively (Yan et al., 2016).

The loci on Mrg06 were identified only in the multi-environment model (Figure 3C). As mentioned previously for the QTL on Mrg02, both regions possibly represent the same QTL due to chromosome rearrangements. Esvelt Klos et al. (2016) identified two different regions influencing heading date on Mrg06, associated to markers GMI_GBS_24858 (45.2 cM) and avgbs_15433 (67.6 cM). Marker avgbs_15433 is only 4.4 cM apart from marker avgbs_cluster_42139.1.55, which was identified in our study (Table 1), suggesting that they are associated to the same genomic region. On the other hand, marker avgbs_220423.1.53 (14.5 cM on Mrg06) may represent a new region associated to heading date in oats. Similarly, a consistent QTL was found on Mrg05 (avgbs_110804.1.56 at 56.5 cM), regardless of the environment and mapping model (Figures 3A-3C and Table 1). To our knowledge, this is the first report of this region controlling heading date in oats. This region may be specific for subtropical-adapted germplasm or was not identified in spring oats.

Studies on QTL mapping in heading date have been conducted on oats, including germplasm adapted to subtropical conditions (Locatelli et al., 2006; Nava et al., 2012; Zimmer et al., 2018). However, these studies used exclusively biparental mapping populations. In this sense, some of these QTL are possibly germplasmspecific. In the present study, we assessed a large set of unrelated oat genotypes in two contrasting subtropical environments, allowing the detection of important regions associated to heading date. Our results showed 
genomic regions controlling oat heading date on Mrg02, Mrg05, Mrg06, Mrg12, and Mrg21. Some of these regions are well-known, such as regions on Mrg02 (Locatelli et al., 2006; Esvelt Klos et al., 2016; Sunstrum et al., 2019; Zimmer et al., 2018), Mrg06 (Esvelt Klos et al., 2016), Mrg12 (Nava et al., 2012; Esvelt Klos et al., 2016; Sunstrum et al., 2019), and Mrg21 (Esvelt Klos et al., 2016; Sunstrum et al., 2019). Mrg02 and Mrg12 regions are associated to Vrn3 gene (Esvelt Klos et al., 2016; Nava et al., 2012), while Mrg21 region is associated to Vrn1 (Esvelt Klos et al., 2016; Maloney et al., 2011). The QTL on Mrg05 is a new genomic region associated to heading date in oats and may be associated exclusively to subtropical germplasm. Access to an annotated oat genome could help identify candidate genes within this region responsible for heading date, but current resources allocated to genomic are limited. Our results provide a strong basis for MAS in oats, allowing selection for heading date in early generations using molecular breeding.

\section{Acknowledgments}

This study was supported by the Coordination for the Improvement of Higher Education Personnel (CAPES), National Council for Scientific and Technological Development (CNPq), and Rio Grande do Sul State Research Support Foundation (PRONEX/FAPERGS; grant number 16/0484-6). The first author was recipient of CNPq (process number 140273/2016-6) and CAPES (process number 88881.187633/2018-01) PhD fellowships. The authors are thankful to the PepsiCo Agro \& Discovery Laboratory/University of Minnesota for supporting the genotyping-by-sequencing. A special thanks to professor Stephen Harrison (Louisiana State University), David Eickholt (PepsiCo), and Mandy Waters (PepsiCo) for collaborating with this research.

\section{Authors' Contributions}

Conceptualization: Zimmer, C.M.; Pacheco, M.T.; Federizzi, L.C.; Data acquisition: Zimmer, C.M.; Oliveira, G.; Pacheco, M.T; Arruda, K.M.A.; Data analysis: Zimmer, C.M.; Design of methodology: Zimmer, C.M.; Pacheco, M.T.; Federizzi, L.C.; Writing and editing: Zimmer, C.M.; Oliveira, G.; Pacheco, M.T.; Arruda, K.M.A.; Federizzi, L.C.

\section{References}

Andrews, S. 2010. FastQC: a quality control tool for high throughput sequence data. Available at: http://www. bioinformatics.babraham.ac.uk/projects/fastqc. [Accessed Oct $15,2018]$

Asoro, F.G.; Newell, M.A.; Scott, M.P.; Beavis, W.D.; Jannink, J.L. 2013. Genome-wide association study for beta-glucan concentration in elite North American oat. Crop Science 53: 542-553.
Bekele, W.A.; Wight, C.P.; Chao, S.; Howarth, C.J.; Tinker, N.A. 2018. Haplotype-based genotyping-by- sequencing in oat genome research. Plant Biotechnology Journal 16: 1452-1463.

Benjamini, Y.; Hochberg, Y. 1995. Controlling the false discovery rate: a practical and powerful approach to multiple testing. Journal of the Royal Statistical Society 57: 289-300.

Bolger, A.M.; Lohse, M.; Usadel, B. 2014. Trimmomatic: a flexible trimmer for Illumina sequence data. Bioinformatics 30: 21142120.

Chaffin, A.S.; Huang, Y.F.; Smith, S.; Bekele, W.A.; Babiker, E.; Gnanesh, B.N.; Foresman, B.J.; Blanchard, S.G.; Jay, J.J.; Reid, R.W.; Wight, C.P.; Chao, S.; Oliver, R.; Islamovic, E.; Kolb, F.L.; McCartney, C.; Fetch, J.W.M.; Beattie, A.D.; Bjørnstad, Å.; Bonman, J.M.; Langdon, T.; Howarth, C.J.; Brouwer, C.R.; Jellen, E.N.; Esvelt Klos, K.; Poland, J.A.; Hsieh, T.F.; Brown, R.; Jackson, E.; Schlueter, J.A.; Tinker, N.A. 2016. A consensus map in cultivated hexaploid oat reveals conserved grass synteny with substantial subgenome rearrangement. Plant Genome 9: 102.

Endelman, J.B. 2011. Ridge regression and other kernels for genomic selection with $\mathrm{R}$ package rrBLUP. Plant Genome 4: 250-255

Esvelt Klos, K.; Huang, Y.F.; Bekele, W.A.; Obert, D.E.; Babiker, E.; Beattie, A.D.; Bjørnstad, Å.; Bonman, J.M.; Carson, M.L.; Chao, S.; Gnanesh, B.N.; Griffiths, I.; Harrison, S.A.; Howarth, C.J.; Hu, G.; Ibrahim, A.; Islamovic, E.; Jackson, E.W.; Jannink, J.L.; Kolb, F.L.; McMullen, M.S.; Fetch, J.M.; Murphy, J.P.; Ohm, H.W.; Rines, H.W.; Rossnagel, B.G.; Schlueter, J.A.; Sorrels, M.E.; Wight, C.P.; Yan, W.; Tinker, N.A. 2016. Population genomics related to adaptation in elite oat germplasm. Plant Genome 9: 103.

Esvelt Klos, K.; Yimer, B.A.; Babiker, E.M.; Beattie, A.D.; Bonman, J.M.; Carson, M.L.; Chong, J.; Harrison, S.A.; Ibrahim, A.M.H.; Kolb, F.L.; McCartney C.A.; McMullen, M.; Fetch, J.M.; Mohammadi, M.; Murphy, J.P.; Tinker, N.A. 2017. Genome-wide association mapping of crown rust resistance in oat elite germplasm. Plant Genome 10: 107.

Federizzi, L.C.; Bertagnolli, P.F.; Carvalho, F.I.F. 1996. Genetics of flowering time in oat (Avena sativa L.). Journal of Genetics and Breeding 50: 147-154.

Holland, J.; Portyanko, V.; Hoffman, D.; Lee, M. 2002. Genomic regions controlling vernalization and photoperiod responses in oat. Theoretical and Applied Genetics 105: 113-126.

Locatelli, A.B.; Federizzi, L.C.; Milach, S.C.K.; Wight, C.P.; Molnar, S.J.; Chapados, J.T.; Tinker, N.A. 2006. Loci affecting flowering time in oat under short-day conditions. Genome 49: 1528-1538.

Maloney, P.V.; Lyerly, J.H.; Wooten, D.R.; Anderson, J.M.; Livingston III, D.P.; Brown-Guedira, G.; Marshall, D.; Murphy, J.P. 2011. Marker development and quantitative trait loci in a fall-sown oat recombinant inbred population. Crop Science 51: 490-502.

Mazurkievicz, G.; Ubert, I.P.; Krause, F.A.; Nava, I.C. 2019. Phenotypic variation and heritability of heading date in hexaploid oat. Crop Breeding and Applied Biotechnology 19: 436-443.

McNish, I.G.; Zimmer, C.M.; Susko, A.Q.; Heuschele, D.J.; Tiede, T.; Case, A.J.; Smith, K.P. 2020. Mapping crown rust resistance at multiple time points in elite oat germplasm. Plant Genome 13: 7 . 
Nava, I.C.; Wight, C.P.; Pacheco, M.T.; Federizzi, L.C.; Tinker, N.A. 2012. Tagging and mapping candidate loci for vernalization and flower initiation in hexaploid oat. Molecular Breeding 30: 1295-1312.

Newell, M.A.; Asoro, F.G.; Scott, M.P.; White, P.J.; Beavis, W.D.; Jannink, J.L. 2012. Genome-wide association study for oat (Avena sativa L.) beta-glucan concentration using germplasm of worldwide origin. Theoretical and Applied Genetics 125: 1687-1696.

O'Donoughue, L.S.; Sorrells, M.E.; Tanksley, S.D.; Autrique, E.; Deynze, A.V.; Kianian, S.F.; Phillips, R.L.; Rines, H.W.; Rayapati, P.J.; Lee, M.; Penner, G.A.; Fedak, G.; Molnar, S.J.; Hoffman, D.; Salas, C.A. 1995. A molecular linkage map of cultivated oat. Genome 38: 368-380.

Poland, J.A.; Brown, P.J.; Sorrells, M.E.; Jannink, J.L. 2012. Development of high-density genetic maps for barley and wheat using a novel two-enzyme genotyping-by-sequencing approach. Plos One 7: e32253.

Sunstrum, F.G.; Bekele, W.A.; Wight, C.P.; Yan, W.; Chen, Y.; Tinker, N.A. 2019. A genetic linkage map in southern-by-spring oat identifies multiple quantitative trait loci for adaptation and rust resistance. Plant Breeding 138: 82-94.
Tinker, N.A.; Bekele, W.A.; Hattori, J. 2016. Haplotag: software for haplotype-based genotyping-by-sequencing analysis. G3: Genes, Genomes, Genetics 6: 857-863.

Yan, H.; Bekele, W.A.; Wight, C.P.; Peng, Y.; Langdon, T.; Latta, R.G.; Fu, Y.B.; Diederichsen, A.; Howarth, C.J.; Jellen, E.N.; Boyle, B.; Wei, Y.; Tinker, N.A. 2016. High-density marker profiling confirms ancestral genomes of Avena species and identifies D-genome chromosomes of hexaploid oat. Theoretical and Applied Genetics 129: 2133-2149.

Zadoks, J.C.; Chang, T.T.; Konzak, F.C. 1974. A decimal code for the growth stages of cereals. Weed Research 14: 415-421.

Zimmer, C.M.; Ubert, I.P.; Pacheco, M.T.; Federizzi, L.C. 2018. Molecular and comparative mapping for heading date and plant height in oat. Euphytica 214: 101.

Zimmer, C.M.; McNish, I.G.; Klos, K.E.; Oro, T.; Arruda, K.M.A.; Gutkoski, L.C.; Pacheco, M.T.; Smith, K.P.; Federizzi, L.C. 2020. Genome-wide association for $\beta$-glucan content, population structure, and linkage disequilibrium in elite oat germplasm adapted to subtropical environments. Molecular Breeding 40: 103. 